\title{
Estudio prospectivo de cinco años de los niveles de homocisteinemia y riesgo cardiovascular en pacientes de una unidad de hemodiálisis
}

\author{
Mateo Párraga Díaz, Esperanza Melero Rubio, María Paz Gómez Sánchez \\ Hospital Clínico Universitario Virgen de la Arrixaca. Murcia
}

\begin{abstract}
Resumen
La enfermedad cardiovascular es compleja y multifactorial. Uno de los marcadores conocido como factor de riesgo vascular independiente es la homocisteína; el aumento en $5 \mu \mathrm{mol} / \mathrm{l}$ por encima del rango normal equivale, en riesgo vascular, a un aumento de $20 \mathrm{mg} /$ dl por encima del colesterol normal. En la población general niveles ligeramente elevados de homocisteína $(>15 \mu \mathrm{mol} / \mathrm{l})$, se asocian al incremento de la mortalidad y de los eventos cardiovasculares. Este factor también está presente en pacientes renales, siendo sus niveles 3-4 veces superiores a la población en general. Los mecanismos por los que la homocisteína está elevada en la insuficiencia renal no están claros, ya que su excreción renal sólo representa el $1 \%$ de su eliminación; si embargo, si está comprobado que la utilización de membranas de diálisis de alto flujo, diálisis lenta nocturna, o hemodiafiltración on-line diarias reducen significativamente los niveles de este aminoácido. Tal vez estos tratamientos, al conseguir una mayor reducción de los niveles de homocisteína, puedan reducir la morbimortalidad cardiovascular renal $y$, por ello, es aconsejable medir los niveles de homocisteína anualmente en los pacientes renales. Nos planteamos conocer el riesgo vascular de nuestros pacientes renales a lo largo de cinco años, según valores de homocisteína y determinar su relación con modalidad y tiempo en tratamiento, edad, sexo y grupo étnico. Realizamos un estudio observacional prospectivo en pacientes de una unidad renal durante cinco años. Recogimos datos sobre: edad, sexo, grupo
\end{abstract}

Correspondencia:

Mateo Párraga Díaz

Unidad Nefro-Diálisis. H.C.U. Virgen de la Arrixaca

Ctra. Madrid-Cartagena, s/n. 30120 El Palmar. Murcia

E-mail:mateo.parraga@carm.es étnico, modalidad de tratamiento (hemodiafiltración versus hemodiálisis), tiempo en tratamiento y valores de homocisteína. Estudiamos 115 pacientes, de edad media 59 años, 68 hombres y 47 mujeres; 107 pacientes caucásicos mediterráneo, 4 caucásicos norteafricano y 4 negroide. El 54,8\% llevaba menos de 4 años en tratamiento renal. Un $63 \%$ estaba en hemodiálisis, $27 \%$ en hemodiafiltración alterna y $10 \%$ en diaria. El valor medio de homocisteína en varones fue de $23.47 \mu \mathrm{mol} / \mathrm{l}$ y en mujeres $24.29 \mu \mathrm{mol} / \mathrm{l}$. Los valores de homocisteína en el tercer y cuarto año de estudio según el grupo cultural fueron en pacientes negroides 51,50 y $69,35 \mu \mathrm{mol} / \mathrm{l}$ respectivamente, con significación estadística. En la población general el riesgo cardiovascular se asocia a la edad y el sexo sin embargo, los niveles de homocisteína versus estas variables en nuestra población renal a estudio no se ven modificados. Otras diferencias vienen marcadas por el grupo étnico: menores en raza negra y asiáticos que en personas de raza blanca, mientras que los latinoamericanos tenían concentraciones intermedias. Estas afirmaciones no coinciden con los resultados de nuestro estudio, ya que el grupo caucásico mediterráneo (la mayoría de nuestros pacientes) que presenta valores intermedios con riesgo moderado y el grupo de etnia negroide valores más elevados incluso de riesgo elevado. Concluimos que nuestros pacientes presentaban un nivel moderado de riesgo vascular. Al enfrentar homocisteína con sexo, edad, modalidad y tiempo de tratamiento sustitutivo no encontramos relación estadística significativa. Sin embargo, el grupo étnico si presentaba variaciones significativas siendo el grupo negroide el de mayor riesgo vascular con niveles más elevados en los tres últimos años del estudio.

\section{PALABRAS CLAVE}

- HOMOCISTEINA

- PROCEDIMIENTO DE DIÁLISIS

- RIESGO CARDIOVASCULAR 


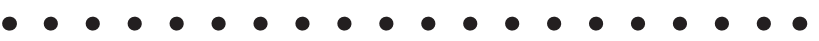

A prospective study of five years about homocysteinemia levels and cardiovascular risk in patients in a hemodialysis unit

\section{Abstract}

Cardiovascular disease is complex and multifactorial. One of the markers known as independent vascular risk factor is homocysteine. In relation to vascular risk, the increase of $5 \mathrm{mmol} / \mathrm{I}$ above the normal range, corresponds to an increase of $20 \mathrm{mg} / \mathrm{dl}$ above normal cholesterol. In the general population, slightly elevated homocysteine levels (> 15 $\mu \mathrm{mol} / \mathrm{I}$ ), are associated with increased mortality and cardiovascular events. Also, this factor is present in renal patients, at 3-times higher levels than the general population. Mechanisms by which homocysteine is elevated in renal failure are unclear, since renal excretion represents only $1 \%$ of its elimination. However, it has been shown that the use daily of membranes of high flux dialysis, slow nocturnal home dialysis, or on-line hemodiafiltration, significantly reduce these amino acid levels. These treatments could achieve a greater reduction in homocysteine levels and reduce the cardiovascular renal morbid-mortality, therefore, it is advisable to measure homocysteine levels in renal patients annually. The aim was to study the vascular risk in our renal patients in a study of five years, according to the homocysteine levels and determine their relationship with modality and time of treatment, age, sex and ethnicity. A prospective observational study of five year was carried out in our dialysis unit. Data about age, sex, ethnicity, type of treatment (hemodiafiltration versus hemodialysis), duration of treatment and homocysteine levels were collected. 115 patients (68 men and 47 women) with a mean age of 59 years were studied. 107 patients were Mediterranean Caucasians, 4 North African Caucasian and 4 African blacks. $54.8 \%$ had less than four years in renal treatment. $63 \%$ were on hemodialysis, $27 \%$ patients were in alternating hemodiafiltration, and $10 \%$ in daily hemodialysis. The mean level of homocysteine in males was $23.47 \mathrm{mmol} / \mathrm{l}$, and in women of $24.29 \mathrm{mmol} / \mathrm{l}$. According to the cultural group, homocysteine levels in the third and fourth year of study, were significant in African black patients, with levels of 51.50 and $69.35 \mathrm{~mol} / \mathrm{I}$ respectively. In the general population, cardiovascular risk is associated with age and sex. However, homocysteine levels versus these variables in our renal study population are not modified. Other differences are marked by ethnic group: lower in blacks and Asians than in Caucasians, while Latin American had intermediate concentrations. This information do not coincide with our findings, because the Caucasian Mediterranean group (most of our patients) had intermediate values at moderate risk and the African black group had higher values, even of the high risk. We conclude that our patients had a moderate level of vascular risk. When relating homocysteine with sex, age, mode and time of replacement therapy no statistical significant relationship was found. However, ethnicity if present significant variations being the African black the group with higher vascular risk with higher levels in the last three years of the study.

\section{KEYWORDS}

- HOMOCYSTEINE

- DIALYSIS PROCESS

- CARDIOVASCULAR RISK.

\section{Introducción}

La enfermedad cardiovascular es compleja y multifactorial y es la mayor causa de muerte en los países industrializados ${ }^{1}$. El elevado riesgo de mortalidad por enfermedad cardiovascular no se puede explicar totalmente por los factores de riesgo tradicionales como hiperlipidemia, tabaquismo e hipertensión ${ }^{2}$; en la actualidad, se relaciona el desarrollo de esta enfermedad con la homocisteina como factor independiente ${ }^{3,4}$. EI aumento de $5 \mu \mathrm{mol} / \mathrm{l}$ de homocisteinemia por encima de su rango normal, equivale en riesgo vascular a un aumento de $20 \mathrm{mg} / \mathrm{dl}$ por encima de la colesterolemia normal5,6. En la población general se ha demostrado que, niveles ligeramente elevados de homocisteína $(>15 \mu \mathrm{mol} / \mathrm{l})$, están asociados con un incremento de la mortalidad y de los eventos cardiovasculares ${ }^{7}$.

La homocisteína es un aminoácido sulfurado, derivado de la metionina y la cistina cuyo metabolismo depende de la vitamina B12 y del ácido fólico, el aumento de homocisteína se debe tanto a causas ambientales (nutricionales y el estilo de vida) como genéticas ${ }^{8}$. Hasta el momento no se conoce con exactitud cómo la hiperhomocisteinemia contribuye al desarrollo de la enfermedad cardiovascular, ni cuáles son los factores que desencadenan el aumento de homocisteína observado en estos pacientes pero si se ha puesto de manifiesto que unos niveles sanguíneos elevados de homocisteína pueden aumentar el riesgo de que una persona padezca una enfermedad cardiovascular, en particular una enfermedad cardíaca. La homocisteína está implicada en el desarrollo de la arteriosclerosis ${ }^{1}$. También hace que las plaquetas se vuelvan más adhesivas favoreciendo la formación de coágulos. Algunos científicos creen que la homocisteína también reduce la flexibi- 
lidad de las arterias y de las venas, impidiendo que se dilaten cuando lo necesitan. Además, la homocisteína reduciría la producción de óxido nítrico y participaría junto con el colesterol en la conformación de la placa de ateroma ${ }^{9,10}$.

Este factor también está presente en pacientes renales ${ }^{11}$, aunque, los mecanismos por los que la homocisteína está elevada en la IRC no están aclarados, ya que su excreción renal sólo representa el $1 \%$ de su eliminación ${ }^{1,12}$. En pacientes en HD los niveles de homocisteína están muy elevados (3-4 veces superiores a la población general) y la prevalencia de hiperhomocisteinemia ${ }^{10}$ alcanza el $85-90 \%$. Aunque descienden después del trasplante renal la prevalencia de hiperhomocisteinemia en estos pacientes también es elevada ${ }^{13}$ (50-75\%). La utilización de membranas de diálisis de alto flujo, la diálisis lenta nocturna, o la HD o HDF en línea diarias, reducen significativamente los niveles de este aminoácido ${ }^{12}$.

Hay que recordar que la relevancia de este estudio está en que la mayor causa de mortalidad en pacientes renales está relacionada con problemas cardiovasculares14. De ahí, la importancia de la monitorización de este marcador para la prevención tales eventos en el paciente renal.

Con el propósito de analizar la relevancia de este factor en los eventos cardiovasculares de nuestros pacientes nos planteamos los siguientes objetivos:

(1) Conocer el riesgo vascular de nuestros pacientes, teniendo como marcador la homocisteína.

(2) Determinar si existe relación entre los niveles de homocisteína y el procedimiento de diálisis utilizado.

\section{Material y método}

Realizamos un estudio en el que se incluyeron a todos los pacientes de nuestra unidad que recibían tratamiento sustitutivo con HDF en línea, alterna o diaria, y HD convencional; comenzando el periodo de seguimiento en mayo de 2009 y finalizando en mayo de 2013.

Se recogieron datos de:

- Edad en años, dividida en cinco periodos: $1^{\text {er }}$ periodo pacientes con edades comprendidas entre 20 y 34 años, $2^{\circ}$ periodo entre 35 y 49 años, $3^{\text {er }}$ periodo entre 50 y 64 años, $4^{\circ}$ periodo entre 65 y 79 años y $5^{\circ}$ periodo igual o mayor de 80 años.

- Sexo: Hombre (H), Mujer (M).
- Grupo cultural o étnico: caucásico mediterráneo, caucásico norteafricano y negroide.

- Tiempo de tratamiento en diálisis en años, dividido en seis periodos: $1^{\text {er }}$ periodo menos de 2 años, $2^{\circ}$ periodo

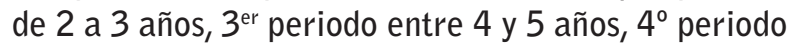
entre 6 y 7 años, $5^{\circ}$ periodo entre 8 y 10 años y $6^{\circ}$ periodo más de 10 años.

- Relacionados con procedimiento dialítico: HDF en línea alterna o diaria y HD.

- Valores analíticos de homocisteína sérica coincidiendo con la analítica anual programada según protocolo de seguimiento a pacientes de nuestra unidad. La muestra se extrajo prediálisis de la conexión aguja arterial, con el paciente sentado en sillón para tratamiento renal.

Agrupamos en cuatro categorías los valores para homocisteína de menos a más riesgo en cuatro rangos 0 intervalos: < de $15 \mu \mathrm{mol} / \mathrm{l}$ (sin riesgo vascular), entre 15 y $30 \mu \mathrm{mol} / \mathrm{l}$ (riesgo moderado), entre 30 y 100 $\mu \mathrm{mol} / \mathrm{l}$ (riesgo elevado) y $>100 \mu \mathrm{mol} / \mathrm{l}$ (riesgo severo).

Para el análisis estadístico se utilizó el programa SPSS 19.0 para Windows. Las variables cuantitativas se expresan en medias y las cualitativas en porcentajes, como método de contraste se utilizan: $T$ de Student, $\mathrm{X}^{2}$ de Pearson y prueba de Anova para datos apareados.

\section{Resultados}

El total de pacientes estudiados fue de 115 , con una edad media de 58,89 \pm 16 años; $68(59,1 \%)$ hombres y $47(40,9 \%)$ mujeres. De ellos, 107 (93\%) era de grupo étnico caucásico mediterráneo, caucásico norteafricano 4 pacientes $(3,5 \%)$ y $4(3,5 \%)$ de etnia negroide.

Del total de los pacientes el $54,8 \%$ de llevaba en tratamiento sustitutivo renal entre menos de laño y 4 años; la distribución tiempo de tratamiento era de $27,8 \%$ menos de dos años, $27 \%$ de 2 a 4 años, 19,1\% de 4 a 6 años, $9,6 \%$ de 6 a 8 años, $6,1 \%$ de 8 a 10 años y $0,9 \%$ más de 10 años de tratamiento. La distribución de los pacientes según los intervalos de edad fue: de 20 a 34 años un 6,10\%, entre 35 y 49 años un $31 \%$, entre 50 y 64 años $23,50 \%$, entre 65 y 79 años $26,90 \%$ y $8,70 \%$ de pacientes con edad igual o mayor de 80 años.

El número de pacientes en cada una de las técnicas de tratamiento sustitutivo es variable (Tabla 1) a lo largo de los años, siendo en su conjunto tratados en HD convencional el $64,43 \%$, en HDF alterna $23,71 \%$ y en HDF diaria $11,86 \%$ de los pacientes. Al relacionar con los rangos establecidos según los años no observamos variaciones en los valores con significación estadística. 
Tabla 1. Distribución de pacientes según tipo de TRS y rango de homocisteina.

\begin{tabular}{|c|c|c|c|c|c|c|c|c|c|c|c|c|c|c|c|}
\hline \multirow[t]{2}{*}{ RANGO HOMOCISTEINA } & \multicolumn{5}{|c|}{$\begin{array}{l}\text { HD CONVENCIONAL } \\
\text { años }\end{array}$} & \multicolumn{5}{|c|}{$\begin{array}{c}\text { HDF ONLINE ALTERNA } \\
\text { años }\end{array}$} & \multicolumn{5}{|c|}{$\begin{array}{l}\text { HDF ONLINE DIARIA } \\
\text { años }\end{array}$} \\
\hline & 1 & 2 & 3 & 4 & 5 & 1 & 2 & 3 & 4 & 5 & 1 & 2 & 3 & 4 & 5 \\
\hline$<15 \mu \mathrm{mol} / \mathrm{l}$ & - & 4 & 5 & 2 & 10 & - & 1 & 1 & 4 & 1 & 2 & 1 & 2 & 1 & 2 \\
\hline$\geq 15 \mathrm{y}<30 \mu \mathrm{mol} / \mathrm{I}$ & 12 & 12 & 12 & 19 & 19 & 6 & 4 & 4 & 8 & 9 & 4 & 1 & 3 & 4 & 3 \\
\hline$\geq 15$ y $<100 \mu \mathrm{mol} / \mathrm{l}$ & 2 & 4 & 6 & 9 & 8 & 1 & 1 & 2 & 4 & - & - & - & - & - & - \\
\hline$\geq 1000 \mu \mathrm{mol} / \mathrm{l}$ & - & - & - & - & - & - & 1 & - & - & - & - & - & - & - & - \\
\hline TOTAL PACIENTES & 14 & 20 & 23 & 30 & 38 & 7 & 6 & 7 & 16 & 10 & 6 & 2 & 5 & 5 & 5 \\
\hline
\end{tabular}

En cuanto al valor de homocisteina media relacionado con las variables demográficas de edad (Figura 1) y sexo (Tabla 2) a lo largo de los cinco años de estudio tampoco encontramos modificaciones estadísticamente significativas; con la variable de grupo étnico (Figura 2), los últimos tres años presentó riesgo elevado con valores de $51,50 \%, 69,35 \%$ y $40,95 \%$ sucesivamente con diferencias significativas respecto al resto de grupos.

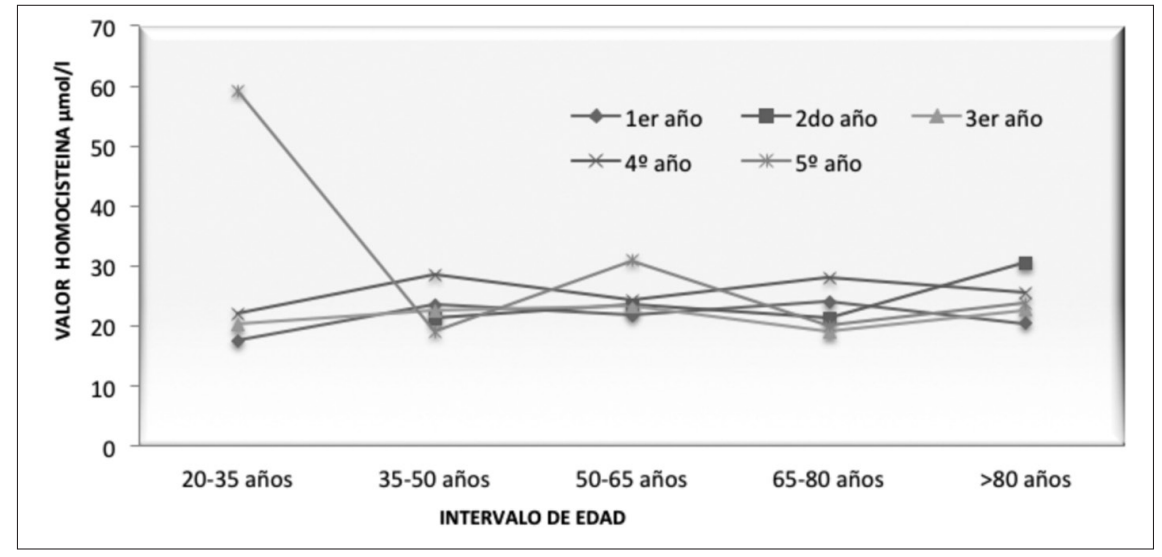

Figura 1. Evolución homocisteina media según intervalo de edad.

Tabla 2. Valor homocisteina media según sexo y año.

\begin{tabular}{c|cccccc} 
& Homcisteínal & Homcisteína2 & Homcisteína3 & Homcisteína4 & Homcisteína5 \\
\cline { 2 - 7 } VARÓN & $22,22 \pm 6,51$ & $23,10 \pm 9,39$ & $22,82 \pm 11,70$ & $27,19 \pm 10,57$ & $22,04 \pm 15,07$ \\
\hline MUJER & $23,70 \pm 4,51$ & $22,82 \pm 8,77$ & $21,69 \pm 11,71$ & $25,55 \pm 16,09$ & $27,68 \pm 28,89$ \\
\hline
\end{tabular}

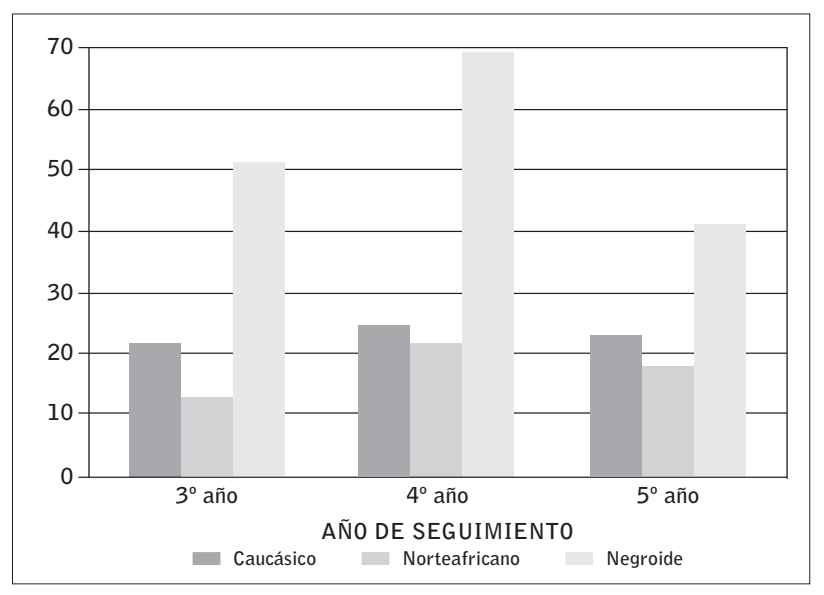

Figura 2. Homocisteina media según etnia o grupo cultural.

\section{Discusión}

En las revisiones realizadas, las variables demográficas modulaban los valores de homocisteina y, por ello, el análisis de estos determinantes (edad, sexo y grupo étnico) ha centrado buena parte de nuestro estudio, así como el procedimiento de diálisis elegido. Resaltamos ciertos aspectos.

Los niveles medios de homocisteina por año relacionados con el rango de edad de los pacientes renales no se vieron modificados durante los cinco años de estudio. Sin embargo, el riesgo cardiovascular en la población en general si se asocia a la edad y el sexo $0^{15,16}$. 
Al relacionar los valores de homocisteina según sexo las medias apenas variaban $1 \mu \mathrm{mol} / /$ entre hombres y mujeres y se mantenía estable a lo largo de todo el periodo. Si nos fijamos en la población en general, hay una diferencia de unos $2 \mu \mathrm{mol} / \mathrm{l}$ menos de homocisteina en la etapa de los 40 años entre el sexo femenino y masculino; posteriormente y a medida que se eleva la edad sobre los 60 años (período posmenopáusico) aumenta la homocisteína en las mujeres tendiendo a igualarse, hecho que puede relacionarse con la filtración glomerular los déficit vitamínicos y la mala absorción intestinal16.

En nuestro estudio la mayoría de los pacientes eran de raza blanca caucásica (93\%), frente a otras razas $7 \%$ (negros 3,5\%, norteafricanos 3,5\%) siendo el grupo caucásico mediterráneo el que presentaba valores intermedios con riesgo moderado y el grupo de etnia negroide el de valores de riesgo elevado en los últimos tres años del estudio con significación estadística. Estos datos no se corresponden con otros estudios donde las concentraciones de homocisteína son menores en raza negra y asiáticos que en personas de raza blanca, mientras que los latinoamericanos tienen concentraciones intermedias ${ }^{16}$. Es obvio que el número de pacientes diferentes a los de etnia caucásica es tan limitado que de los resultados no se pueden extraer conclusiones.

Por último, en lo que respecta a la modalidad o procedimiento de diálisis tampoco encontramos diferencias significativas entre ellas y los valores de homocisteína, pero si observamos una tendencia a mantener niveles moderados en todas las técnicas siendo remarcable que no había ningún paciente que estuviese en HDF en línea diaria con valores intermedios o severos. Estos resultados se asemejan a algunos estudios donde los niveles de homocisteína en pacientes en HDF son significativamente inferiores a los niveles de los pacientes en tratamiento con HD ${ }^{12,16-18}$ (hecho sorprendente pues la excreción de homocisteína a nivel renal sólo representa un $1 \%$ ).

Un aspecto muy relacionado con los eventos vasculares y con el tratamiento renal es el estado del acceso vascular (AV). Un estudio más profundo de los datos aquí presentados nos ayudaría a obtener algunas repuestas sobre una de las causas de la disfunción del $\mathrm{AV}$, la trombosis y, con ello, detectarla prematuramente o prevenirla.

A la vista de los resultados expuestos concluimos que no encontramos diferencias significativas en los niveles de homocisteína según el tipo de tratamiento dia- lítico, nuestros pacientes presentan un nivel moderado de riesgo vascular con una tendencia a mantener niveles séricos a lo largo de los años. Sin embargo, si encontramos diferencias significativas en los pacientes del grupo étnico tipo negroide que presentan riesgo vascular elevado mantenido.

Por otro lado, al enfrentar el nuevo marcador de riesgo vascular con otros factores como edad, sexo y tiempo desde el inicio de programa de tratamiento renal sustitutivo observamos que los niveles no varían significativamente.
Recibido: 10 septiembre 2014
Revisado: 14 octubre 2014
Modificado: 14 octubre 2014
Aceptado: 20 octubre 2014

\section{Bibliografía}

1. Bermúdez M, Briceño I, Gil $F$, Bernal J. Homocisteína y polimorfismos de cistationina $B$ sintasa y metilentetrahidrofolato reductasa en población sana de Colombia. Colomb Med. 2006; 37:46-52.

2. Kullo J, Gau GT, Tajik AJ. Novel Risk Factor for Atherosclerosis. Mayo Clinic Procedures. 2000; 75: 369-80.

3. Christen WG, Ajani UA, Glynn RJ, Hennekens CH. Blood Levels of Homocysteine and Increased Risks of Cardiovascular Disease: Causal or Casual? Arch Intern Med. 2000; 160: 422-434.

4. Refsum H, David Smith A, Ueland PM, Nexo E, Clarke $\mathrm{R}, \mathrm{McPartlin} \mathrm{J}$, y cols. Facts and recommendations about total homocysteine determinations: an expert opinion. Clin Chem. 2004; 50: 3-32.

5. Rey RH, Soifer S, Pombo G y col. Nuevos factores de riesgo. Rev Argent Cardiol [en línea] 2001 [fecha de acceso 24 de noviembre de 2005]; 69 (1): 1-14. Disponible en: http://www.sac.org.ar/rac/2001/ suplemento_3/integrantes.pdf http://www.sac.org. ar/rac/2001/suplemento_3/nuevosfactores.pdf

6. Seshadri N, Robinson K. Homocisteína, vitaminas B, y arteriopatía coronaria. Medical Clinics of North America. 2000; 84:219- 4. 
7. Arenas Jiménez D. Actualizaciones en hemodiálisis. Nefrología. 2008; supl 5: 71-6.

8. De Luis DA, Fernández N, Aller R. Homocisteina, metabolismo y determinantes higienicodietéticos. Endocrinol Nutr. 2004; 51 (8): 458-63.

9. Bostom AG, Lathrop L. Hyperhomocysteinemia in End-stage Renal Disease: Prevalence, Etiology, and Potential Relationship to Atherosclerotic Outcomes. Kidney Int. 1997; 52:10-20.

10. Bostom AG, Gohh RY, Liaugaudas G, Beaulieu AJ, Han $H$, Jacques PF, y cols. Prevalence of Mild Fasting Hyperhomocysteinemia in Renal Transplant Versus Coronary Artery Disease Patients After Fortification of Cereal Grain Flour with Folic Acid. Atherosclerosis. 1999; 145: 221-224.

11. Sarnak MJ, Levey AS, Schoolwerth AC, Coresh J, Culleton B, Hamm LL y cols. Kidney Disease as a Risk Factor for Development of Cardiovascular Disease. A Statement from the American Heart Association Councils on Kidney in Cardiovascular Disease, High Blood Pressure Research, Clinical Cardiology, and Epidemiology and Prevention. Hipertensión. 2003; 42: 1050-65.

12. Cases A. Otros factores de riesgo cardiovascular y renal. Hipertrofia del ventrículo izquierdo. Fibrilación auricular. Tabaquismo. Obesidad. Factores emergentes de riesgo cardiovascular: Homocisteína. Proteína C reactiva. Fibrinógeno. Nefrología. 2004; 24 (6): 62-72.
13. Arnadottir M, Hultberg B, Wahlberg J, Fellstrom B, Dimeny E. Serum Total Homocysteine Concentration Before and Alter Renal Transplantation. Kidney Int. 1998; 54: 1380-84.

14. Goicoechea M Á. Enfermedad cardiovascular en pacientes con estadios 4 y 5 de enfermedad renal crónica. Pacientes en diálisis crónica. Nefrología. 2004; 24 (6): 142-160.

15. Millán I, De Alvaro F. Homocisteína y disfunción endotelial en pacientes con diabetes mellitus, insuficiencia renal crónica, diálisis y trasplante. Nefrología. 1998; XVIII (3): 186-195.

16. Melero-Rubio E, Párraga-Díaz $M$, GómezSánchez MP, Pellicer-Villaescusa S, MerchánMayado E. Observational study on makers of cardiovascular risk in renal patients: conventional vs. haemofiltration on line. Journal of Renal Care. 2009; 35 (4): 201-4.

17. Maduell F, Navarro V, Torregrosa E, Rius A, Dicenta F, Cruz MC, Ferrero JA. Change from Three Times a Week On-line Hemodiafiltration to Short Daily On-line Hemodiafiltration. Kidney Int. 2003; 64: 305-13.

18. Galland R, Traeger J, Arkouche W, Cleaud C, Delawari E, Fouque D. Short Daily Hemodialysis Rapidly Improves Nutritional Status in Hemodialysis Patients. Kidney Int. 2001; 60: 1555-60. 